\title{
Exploring Science Education Anxiety Among Early Childhood Teachers Using Q-Methodology
}

\author{
Ja Eun Kim ${ }^{1}$, Yeon Ha Kim² \\ Department of Early Childhood Education, Kyung Hee University, Seoul, Korea ${ }^{1}$ \\ Department of Child \& Family Studies, Kyung Hee University, Seoul, Korea ${ }^{2}$ \\ $\mathrm{Q}$ 방법론을 사용한 유아교사의 과학교육에 대한 불안 탐색 \\ 김자은 ${ }^{1}$, 김연하 ${ }^{2}$ \\ 경희대학교 유아교육과 ${ }^{1}$, 경희대학교 아동가족학과 ${ }^{2}$
}

\begin{abstract}
Objective: This study aimed to explore early childhood educators' anxiety regarding science education via Q-methodology.

Methods: As the first step, $268 \mathrm{Q}$ samples were collected from resources such as books and research papers related to science education for young children. Through five stages of excluding and modifying, a set of $33 \mathrm{Q}$ statements was finalized. The $\mathrm{P}$ sample consisted of 40 early childhood educators and was based on educational levels, years of teaching experiences and types of centers. The collected data were analyzed using QUANL statistical program.

Results: The results of this study indicate that science anxiety among early childhood educators are classified into four types, explaining $45.13 \%$ of the total variance.

Conclusion: We conclude that early childhood educators' science anxiety stems from "preparing educational environment and materials," "instructional methods focused on process and scientific inquiry skills," "lack of understanding regarding early childhood science education," and "simple science avoidance."
\end{abstract}

Keywords: anxiety, early childhood science education, Q-methodology

\section{서론}

급속하게 이루어진 과학의 발전으로 인하여 과학기술이 우 리 생활 전반에 깊숙이 관여하고 있으며, 앞으로 어떤 모습으 로 과학이 우리의 삶을 변화시킬지 예측하기 어려운 상황이 되었다. 모든 사람이 과학자가 되어 과학기술을 개발할 필요 는 없지만, 과학적 태도와 사고를 갖추는 것은 일상생활에서 이성적이고 효율적인 문제해결을 위해 누구에게나 필요하며, 그 기초를 잘 확립할 수 있는 시기가 바로 유아기이다(Perry \&

Corresponding Author: Yeon Ha Kim, Department of Child \& Family Studies, Kyung Hee University, 26, Kyungheedae-ro, Dongdaemun-gu, Seoul, 02447, Korea

E-mail: yeonhakim@khu.ac.kr
Rivkin, 1992). 특히 유아과학교육은 전문교과형식으로 제공 되는 초중등 교육과는 다르게 이루어져야 하는데, 일상 속에 서 호기심을 자극하는 많은 과학적 탐구의 기회를 유아들에 게 제공하여, 과학적 기초 지식과 탐구하는 태도 및 기술을 익 히도록 도와야 한다(Ministry of Health and Welfare [MOHW], 2013).

유아과학교육의 성패를 결정짓는 요소로서 교육의 주체인 유아교사의 역량은 매우 중요하다. 유아교사가 과학에 대해 긍정적 또는 부정적 태도인지 혹은 어느 정도의 과학적 지식

(C)The Korean Association of Child Studies

This is an Open Access article distributed under the terms of the Creative Commons Attribution Non-Commercial License (http:// creativecommons.org/licenses/by-nc/4.0) which permits unrestricted noncommercial use, distribution, and reproduction in any medium, provided the original work is properly cited. 
이 있는지에 따라 교육목표 설정이 달라지며, 나아가서는 교 수의 실제가 좌우될 수 있다. J. H. Cho와 Kim (2013)은 과학교 과에 관한 지식수준이 높은 교사일수록 과학교수에서 적극성 을 가지고 있으며, Kwon (2007)은 과학교수 효능감이 높은 교 사들이 지도하는 유아들의 과학 활동 참여도가 과학교수 효능 감이 낮은 교사들이 지도하는 유아들의 참여도 보다 높음을 보고하였다. 그러나 우리나라 유아교사는 다른 교과 영역에 비해 과학영역의 지도를 회피하거나 자신 없어 한다고 알려져 있다. 예를 들면, Y. H. Kim (2010)은 예비 유아교사들을 대상 으로 유치원 교육과정의 각 생활영역에 대하여 스스로의 교수 역량을 어느 정도 평가하고 있는지 분석하였는데, 탐구생활영 역의 교수역량 지각이 다른 생활영역에 비해 유의하게 낮음을 밝혔다. K. M. Lee (2009)는 유치원 교사 5명의 면담 자료, 과 학자서전, 과학저널, 수업계획안 등을 질적으로 분석한 결과, 유아과학교육은 '부담으로 다가오는' '보여주기 위한' '구색으 로 존재하는' 그래도 '포기할 수 없는 과학교육'으로 유치원 교 사에게 인식되고 있다고 결론 내렸다.

효율적 유아과학교육은 교사를 통해 달성 될 수 있으나 실 제로 우리 유아교사들의 과학교육에 대한 역량과 자신감은 우 려할 만한 수준이라는 학계와 현장의 고민을 반영하듯, 유아 교사들의 과학교수와 관련하여 많은 연구들이 진행되었다. 그 중 가장 빈번하게 탐색된 개념이 유아교사의 과학 교수 효능 감, 유아교사의 과학교과지식, 그리고 과학교수태도이다(B.

K. Cho \& Seo, 2001; H. Kim, 2013; E. J. Lee, 2013). 이 세 변인 들은 상호간에 높은 정적 관련이 있으며(H. Kim, 2013), 교사 의 학력 및 경력과 교사연수 경험 등을 예측요인으로 갖는다 (B. K. Cho \& Seo, 2001). 그러나 유아교사가 과학교육을 꺼려 하고 역량지각을 낮게 한다는 연구결과와는 역설적으로 이러 한 개념들은 모두 유아교사가 과학교육에 있어서 얼마나 유능 한 존재인지를 측정하고 있다.

일부 연구자들은 유아교사의 과학교육과 관련된 불안 을 과학불안(K. M. Lee, 2005), 과학활동불안(Y.-O. Kim, Lee, Cho, \& Cha, 2012) 그리고 과학교수불안(H. Kim \& Chae, 2014; B. K. Cho \& Go, 2004) 등의 변수명으로 직접 다루었다. 이러한 연구의 결과들은 앞서 기술한 연구결과들과 유사한 맥락을 보이는데, 과학교과지식의 수준이 낮거나, 낮은 과학 교수 효능감과 과학교수태도를 가진 교사들은 과학교육과 관 련된 불안을 높게 지각하였다(Y.-O. Kim et al., 2012). 또한 학 력 및 경력이 낮거나 교사연수 경험 혹은 기관의 지원이나 시 설이 부족한 경우가 과학 불안을 어느 정도 예측하였다(B. K. Cho \& Go, 2004). 그러나 이 연구들은 비록 변수 명칭에는 약
간의 차이가 있으나 모두 Science-State Trait Anxiety Inventory (S-STAI; Sherwood \& Westerback, 1983)라는 예비 초등학교 교 사들을 위해 개발된 외국 도구의 한국어 수정판을 사용하고 있다. S-STAI의 한국어 수정판은 과학교육과 관련된 불안을 교수불안(과학수업과 관련하여 느끼는 근심, 두려움, 걱정)과 상태불안(과학에 대하여 일반적으로 가지는 불안)의 두 범주 로 점수화하는 형태로서 유아교사들이 지각하는 과학교수와 관련된 불안에 대한 다양하고 구체적 정보를 제공하지는 못 하고 있다.

또한 기존연구에서 다루어진 효능감, 과학교과지식, 과학 교수태도, 과학불안 등은 모두 자기보고식 자료수집에 의존 하는 공통변량이 상당히 큰 변수들임을 고려해 볼 필요가 있 다. 연구모형에 이러한 변수들을 독립 혹은 종속변인으로 배 치하면 통계적으로는 유의미한 결론을 얻을 수는 있으나 이러 한 결론을 바탕으로 현장에 의미 있는 독창적 제안을 하기는 어렵다. 예로서 유아교사의 과학 교수효능감을 높이고 불안을 줄이는 방편으로 가장 많이 제안되는 것이 직전교육과 연수프 로그램(H. Kim \& Chae, 2014; Y.-O. Kim et al., 2012)이나, 구 체적으로 어떤 교육을 어떻게 설계해야 하는지에 대한 진지한 탐색이 필요한 시점이다.

유아과학교육의 성공을 유아교사를 통해 이끌어내기 위해 서는 현장에서 유아교사가 느끼는 과학교육과 관련된 불안감 을 직접적으로 탐색하고, 이를 유형화한 후에 그에 따른 대안 을 마련하는 것이 효율적이다. 불안이란 인간의 주관성에 기 초를 두고 있기 때문에, 그 탐색방법에 있어 선구조화 된 하위 개념에 동의 정도를 표시하는 양적 방식으로는 그 집단이 가 진 특유의 불안을 알아내기는 어렵다. 통합중심의 교수방법이 강조되는 유아교육 현장에서 전문교과영역으로 인식되는 과 학을 발달의 개인차가 큰 유아들을 대상으로 교육한다는 점은 유아교사들에게만 국한된 특별한 도전이다. 또한 일상생활의 경험을 의미 있는 과학교육으로 연결시켜야 하는 것은 매우 높은 해석능력과 교육적 전문성을 요구한다. 따라서 과학교육 에 자신 없어하는 교사들에게 얼마나 유능한지 자신을 평가하 게 하는 방식이나 상급학교 교사들을 위해 개발된 척도를 수 정하여 사용하는 연구방식에는 한계가 따른다. 이에 본 연구 에서는 유아과학교육에서 유아교사가 느끼는 불안의 유형을 $\mathrm{Q}$ 방법론을 사용하여 새롭게 접근해 보고자 한다.

$\mathrm{Q}$ 방법론이란 여러 학문분야의 개념이 조합된 독특한 연 구 기법으로 새로운 현상 혹은 특정집단의 다양한 관점이나 시각을 확인하는데 유용하다. 전통적 양적 연구는 일반적으로 모집단에서 연구대상자를 추출하고 미리 구조화된 변인에 의 
거하여 인간의 감정과 행동을 수치화 하고 이 변인들 사이의 관계를 검증하거나 요인 분석하여 재구조화 한다. 이에 비해 자아 지시적 주관성 (operant subjectivity) 탐색을 주목적으로 하 는 $\mathrm{Q}$ 방법론은 $\mathrm{Q}$ 모집단에서 $\mathrm{Q}$ 표본(주관성을 나타내는 진 술문)을 추출하고, $\mathrm{P}$ 표본(연구대상자)에게 $\mathrm{Q}$ 표본에 수치를 부여하게 한 후에 이를 토대로 공통적 주관성을 추구하는 사 람들을 요인으로 추출한다. 즉, 집단 구성원이 가진 주관성 속 에서 보편을 이끌어내는 연구방법으로 요약될 수 있으며 양적 연구와 질적 연구의 특성을 공유하고 있다(S. E. Kim, 2010). 이때, Q 모집단은 양적연구의 모집단, $\mathrm{Q}$ 표본은 양적연구의 연구대상자에 해당하고, P 표본은 양적연구의 변인에 해당한 다고 볼 수 있으며, 양적 연구에서와 마찬가지로, $\mathrm{Q}$ 표본이 대
표성 있게 선정되었는지, $\mathrm{P}$ 표본이 타당하게 선정되었는지에 연구의 적절성이 좌우된다(B. J. Kim, 1999).

종합하면, 본 연구는 과학교수와 관련되어 우리의 유아교 사들이 역량 지각을 낮게 하거나 불안해 한다는 선행연구 결 과에 기초하여, 유아교사들이 과학교육과 관련하여 느끼는 불 안을 Q 방법론을 사용하여 탐색하고자 한다. 본 연구는 선구 조화된 개념을 중심으로 양적 측정에 의존하던 기존의 유아교 사의 과학교육과 관련된 교사연구의 패턴에 변화를 추구하고 유아교사의 과학교육과 관련된 불안에 대한 개념을 확립하는 데 일조하는 것이 목표이다. 본 연구가 설정한 연구문제는 다 음과 같다.

Step 1: Selection of Q sample

- Composition of Q population

- Selection of Q sample

- Reliability

- Making Q cards and Q distribution chart

\begin{tabular}{|c|}
\hline $\begin{array}{c}\text { Composition of } \\
\text { Q population }\end{array}$ \\
\hline $\begin{array}{c}268 \text { statements from } \\
\text { academic literature } \\
\text { regarding science for } \\
\text { young children }\end{array}$ \\
\hline
\end{tabular}

\begin{tabular}{|c|}
\hline $\begin{array}{c}\text { Selection of } \\
\text { Q sample }\end{array}$ \\
\hline $\begin{array}{c}\text { Selection of } 33 \\
\text { statements from the } \\
268 \text { Q population }\end{array}$ \\
\hline
\end{tabular}

I

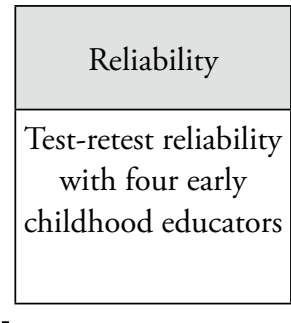

Q cards and Q distribution chart

Creating Q cards and

Q distribution chart for data collection

\section{I}

\begin{tabular}{|l|}
\hline \multicolumn{1}{|c|}{ Step 2: Selection of P sample } \\
\hline \\
\hline \multicolumn{1}{|c|}{$\mathbf{\perp}$ early childhood educators at the Seoul and Gyeonggi-do area } \\
\hline \multicolumn{1}{|c|}{ Step 3: Q sorting } \\
\hline Interviews
\end{tabular}

I

\begin{tabular}{|c|c|}
\hline Q cards classification & Interviews \\
\hline $\begin{array}{c}\text { Classification of Q statements based on } \\
\text { levels of agreement into 9 piles }\end{array}$ & $\begin{array}{c}\text { Asking reasons for strong agreement or } \\
\text { disagreement with specific Q statements }\end{array}$ \\
\hline Step 4: Data analysis \\
\hline - Principal component factor analysis through QUANL Program \\
\hline
\end{tabular}

Figure 1. Research process. 


\section{연구문제 1}

유아교사의 과학교육과 관련된 불안의 인식유형은 어떠한가?

\section{연구방법}

\section{연구절차}

본 연구의 1 단계에서는 $\mathrm{Q}$ 모집단의 구성과 $\mathrm{Q}$ 표본의 선정, 신 뢰도 검증 및 $\mathrm{Q}$ 카드와 분포도를 준비하였다. 다음으로 2 단계 에서는 P 표본을 선정하고, 3 단계에서는 $\mathrm{Q}$ 분류와 심층면담 을 진행하였다. 마지막 4 단계에서는 자료의 처리와 분석을 하 였다. 이러한 연구절차와 과정을 도해하면 Figure 1 과 같다.

\section{1단계: $\mathrm{Q}$ 표본(Q sample)의 선정}

Q모집단(Q population)의 구성 $\mathrm{Q}$ 모집단에 필요한 진술문 작성법은 크게 구술형과 추출형으로 나뉜다. 구술형은 연구자 가 연구와 관련된 사람들을 대상으로 연구의 주제에 대한 인터 뷰를 통해 도출된 결과로 진술문을 작성하는 것이며, 추출형은 연구주제와 관련된 다양한 문헌자료 및 선행연구 등으로부터 추출하는 것이다. 본 연구에서는 추출형의 방법을 사용하였으 며, 유아교사의 과학교육과 관련된 선행연구 및 문헌고찰을 통 해 268개의 Q 모집단을 구성하는 진술문을 추출하였다. 문헌 자료 목록은 Table 1 과 같다.
$Q$ 표본(Q sample) 선정 유아교육관련 현장 전문가 3명(서울 $\mathrm{Y}$ 유치원 교사, 경기도 $S$ 유치원 원장, 경기도 $S$ 유치원 교사)과 학부와 대학원에서 과학교육을 지도하는 대학교수 1 명(서울 $\mathrm{K}$ 대학교)이 총 5 차례 분석과정을 거처 268 개의 $\mathrm{Q}$ 모집단을 33 개의 Q 표본 진술문으로 선정하였다. 진술문의 유사성, 상 호배타성, 포괄성 등을 고려한 분석으로 1 차에서 70 개의 진술 문을 추출하고, 2 차에서는 진술문들이 균형을 이루고 있는지 를 살펴보기 위한 목적으로 범주화 작업을 하였다. 진술문들 은 크게 교사의 과학지식, 교사의 과학교수방법, 학습자의 발 달수준, 과학에 대한 태도 및 가치, 환경구성에 관한 불안으로 범주화되었다. 3 차에서는 범주화한 진술문 중 유사하게 인식 되는 것을 제외하여 41 개의 진술문으로 압축하고, 4 차에서는 재검토 및 통합의 과정을 통해 34 문항으로 재추출하였다. 5 차 에서는 진술문들의 문장을 다듬었고 한 개의 유사 문항을 추 가적으로 제거하여 최종적으로 33 개 Q 표본 진술문을 선정하 였다. 이들 33 개의 Q표본 진술문은 Table 2 와 같다.

$\mathrm{Q}$ 표본에 대한 신뢰도 검사는 4 명에게 시간차를 두고 $\mathrm{Q}$ 분 류를 두 번 실시하였다.

처음 사람에게는 이틀 후 두 번째 $\mathrm{Q}$ 분류를 실시하고, 두 번 째 사람에게는 일주일 간격, 세 번째 사람에게는 열흘간격, 네 번째 사람에게는 2 주 간격을 두고 $\mathrm{Q}$ 분류를 실시하였다. 전체 적으로 주관적 인식에 관한 진술문의 신뢰도는 $r=.84$ 로 나타 나 문항에 대한 반응의 신뢰도 상관계수는 매우 양호하였다. $\mathrm{Q}$ 카드는 선정된 진술문 33개를 연구대상자가 읽기 쉽고 취

Table 1

Reference Data List

\begin{tabular}{|c|c|c|}
\hline Subject & Author & Source \\
\hline Science anxiety & Kang (2007) & Master's thesis \\
\hline \multirow[t]{2}{*}{ Science pedagogical content knowledge } & S. Park (2003) & Journal article \\
\hline & E.-S. Shin \& Kim (2007) & Journal article \\
\hline \multirow[t]{3}{*}{ Science teaching efficacy } & D. Shin (2010) & Master's thesis \\
\hline & H.-S. Park (2007) & Master's thesis \\
\hline & H.-S. Cho (1998) & Journal article \\
\hline \multirow[t]{4}{*}{ Science teaching method } & E.-J. Lee (2010) & Doctoral dissertation \\
\hline & M. A. Kim (2002) & Doctoral dissertation \\
\hline & S. J. Lee (2007) & Master's thesis \\
\hline & M.-A. Lee (2008) & Master's thesis \\
\hline \multirow[t]{4}{*}{ Early childhood science education course } & Ministry of Education and Science Technology (2013) & Book \\
\hline & B. K. Cho, Go, \& Nam (2015) & Book \\
\hline & Hwang \& Cho (2001) & Book \\
\hline & Han (2010) & Book \\
\hline
\end{tabular}


급이 용이하도록 $5 \mathrm{~cm} \times 4 \mathrm{~cm}$ 의 카드에 인쇄하여 제작하였다. $\mathrm{Q}$ 표본의 분포도는 진술문에 대한 의견 동의 정도에 따라 9점 척도 위에 정해진 숫자의 카드를 놓는 강제분류법으로 Figure 2 와 같이 사용하였다.

\section{2단계: P 표본(P sample)의 선정}

$\mathrm{Q}$ 분류에 참여하게 되는 대상자들을 $\mathrm{P}$ 표본(P sample)이라고 하며 Q 방법론에서는 적은 수의 사람을 대상으로 한다. 이러
한 이유는 주관성에 대한 심층적이고 해석적 이해에 중요성 을 두기 때문이다. 다만 P 표본은 연구 주제와 관련된 견해 또 는 경험을 한 사람들을 대상으로 하여야 하며, 집단을 잘 대표 할 수 있도록 고르게 선정한다(H. K. Kim, 2008). 이에 따라 본 연구에서는 서울, 경기지역의 공·사립유치원에 재직하고 있는 유아교사 40 명을 P 표본으로 선정하였다. 이들은 모두 자발적 으로 본 연구 참여에 동의하였으며 가능한 연령, 학력과 경력 이 고르게 분포할 수 있도록 선정하였는데, P 표본의 연령은 만 22세부터 만 53세까지이며, 최종학력은 2,3 년제 전문대졸

Table 2

Q Sample for Early Childhood Science Education Anxiety

\begin{tabular}{lcl}
\hline & No. & Q statements \\
\hline Scientific & 1 & I have difficulty understanding differences between science for children and general science. \\
knowledge of & 2 & I have difficulty clearly understanding scientific inquiry skills for children. \\
teachers & 3 & I feel burdened by learning scientific terms and knowledge. \\
& 4 & I have difficulty understanding cognitive development of children related to science education. \\
& 5 & I have difficulty understanding how the topics of Nature inquiry area in Nuri curriculum are inter-related. \\
& 6 & I have difficulty understanding the linkages between science activities in preschool levels and elementary science \\
& 7 & I am not interested in science and I lack the opportunity to acquire scientific knowledge.
\end{tabular}

Science instructional methods of teachers

Science and developmental levels of children

Attitude and values regarding science for young childrenng

Learning environment regarding science for young children
8 I have difficulty finding adequate questions for developing scientific concepts of young children.

9 I have difficulty providing developmentally appropriate science materials for young children.

10 I have difficulty demonstrating scientific inquiry skills such as observation, test, and prediction.

11 I have difficulty searching proper activities addressing specific science concepts.

12 I have difficulty planning step by step instruction for science activities.

13 I have difficulty integrating science activities across the other educational areas.

14 I have difficulty emphasizing discussion and exchanging ideas in process rather than focusing on results in science activities.

15 I think teach young children science is difficult if they cannot read or write yet.

16 I think science is too difficult for children so that it is hardly teachable.

17 I think children are not interested nor have curiosity in the scientific problems.

18 I think individually appropriate science education for young children is difficult.

19 I have difficulty including children when I plan science activities.

20 I have difficulty including children when I evaluate science activities.

21 I think it is too early to teach science for preschoolers.

22 I think science education happens naturally without careful planning of science activities.

23 I think teaching basic self-help skills are more important than proceeding scientific activities.

24 I think socio-emotional activities are more important than science activities.

25 I think reading, writing and counting activities are more important than science activities.

26 I think developing curiosity in science and inquiry skills are not important for children.

27 I think basic principles regarding object and matter are not important for children.

28 I think basic knowledge regarding object and matter is not important for children.

29 I have difficulty collecting materials and equipment from diverse sources for scientific activities.

30 I am afraid of proceeding science activities because of a lack of scientific equipment in my institution.

31 I am afraid of proceeding science activities because of extra duties and time limits in my institution.

32 I am afraid of proceeding science activities because of difficulties in taking care of animals, plants and science materials.

33 I am afraid of proceeding science activities because my classroom gets noisy and massy. 


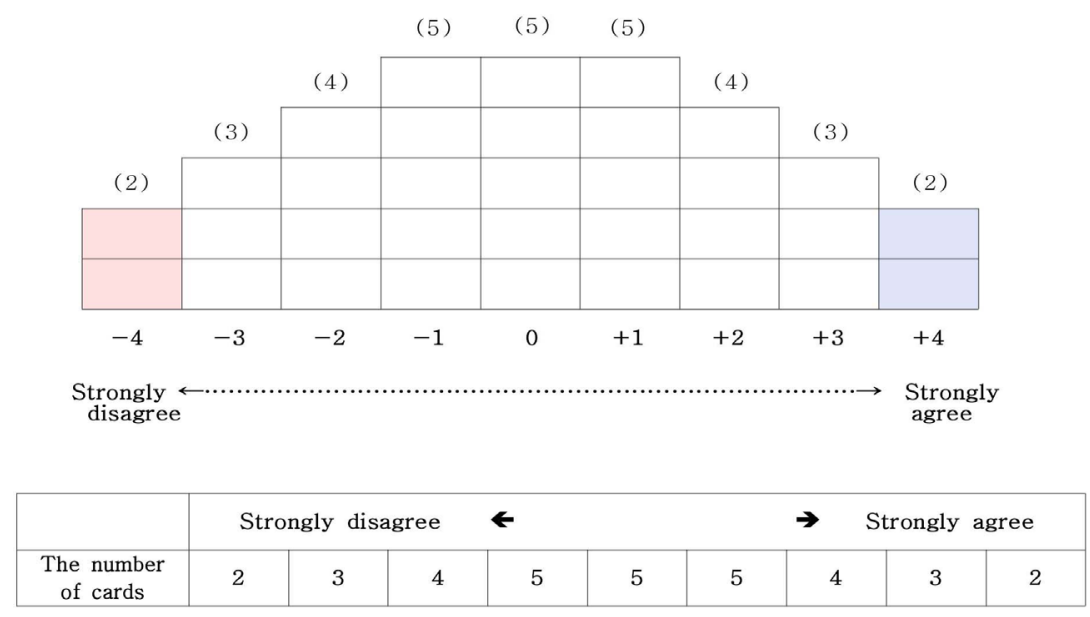

Figure 2. Q sample distribution chart.

16 명, 4년제 대졸 20명, 석사학위 4명이었다. 경력은 만 6개월 부터 만 27년까지 분포되었다.

\section{3 단계: $\mathrm{Q}$ 분류(Q sorting)}

$\mathrm{Q}$ 분류의 구체적인 단계는 먼저 응답자가 33 개의 $\mathrm{Q}$ 카드 진 술문을 읽으면서 전체적인 내용을 파악하고 자신의 주관적인 의견에 따라 동의, 중립, 비동의의 세 그룹으로 진술문 카드를 나눈다. 후에 분포도의 카드 수에 맞추어 카드를 배열하고, 카 드의 위치를 조정하여 $\mathrm{Q}$ 카드의 고유번호를 분포도의 네모 칸에 기록한다. 마지막으로 양극에 놓인 카드 $(-4,+4$ 에 있는 카 드) 즉, '강한 동의'와 '강한 비동의'로 분류한 문장에 대해 응답 자의 분류 이유 및 생각을 자유롭게 서술한다. 본 연구의 제 1 저자가 응답자들의 $\mathrm{Q}$ 분류를 보조하였으며, $\mathrm{Q}$ 분류는 1 인당 대략 약 15-20분이 소요되었다.

\section{4단계: 자료처리 및 분석}

점수화된 자료의 분석은 QUANL program (Korean Society for Scientific Study of Subjectivity, Anseong)을 이용하여 처리하였 으며 $\mathrm{Q}$ 요인 분석은 주요인 분석(principal component factor analysis)방법을 이용하였다. 가장 이상적인 요인수의 결정을 위하여 Eigen value 1.0이상을 기준으로 요인수를 다양하게 입 력시켜 산출된 결과 중 최선이라고 판단된 유형을 선택하였 고, 전체 변량에 대한 요인들의 설명변량이 적당한 것을 고려 한 최선의 요인수가 고려되었다. 각 진술문의 경우에 표준점 수(Z)로 살펴보았다.

\section{연구결과}

\section{과학교육 불안에 대한 유아교사의 인식 유형}

QUANL program을 이용하여 유아교사들이 생각하는 과학 불안에 대한 인식을 Q 요인 분석한 결과 4 개의 유형으로 나 타났다. Table 3에서 나타난 것과 같이 4 개의 유형은 전체변 량의 $45.13 \%$ 를 설명하고 있다. 각 유형별 설명력은 제 1 유형 이 $15.70 \%$, 제 2 유형이 $13.53 \%$, 제 3 유형이 $8.70 \%$, 제 4 유형이 $7.19 \%$ 였다.

연구대상자들은 제 1 유형에 11 명, 제 2 유형에 12 명, 제 3 유형 에 10 명, 제 4 유형에 7 명이 분류되었다. 각 유형별 인자가중치 (factor weight)는 Table 4에서 제시하는 바와 같다. 각각의 유형 내에서 인자가중치가 높을수록 그가 속한 유형에 있어서 그 유형을 대표할 수 있는 전형적(prototype) 혹은 이상적(ideal)인 사람임을 나타낸다.

\section{과학교육 불안에 대한 유아교사의 인식 유형별 특성}

\section{제1유형: 과학 환경구성 및 자료준비에 따른 불 안형}

제 1 유형으로 분류된 대상자는 11 명으로 나타났다. 경력이 5 년 미만인 교사가 6명, 5년-10년 미만인 교사가 3명, 10년 이 상인 교사가 2 명이었고, 기관유형은 국공립유치원 교사가 5 명, 사립유치원 교사가 6 명이었으며, 소지자격은 유치원 2급 정교사가 7 명, 유치원 1 급 정교사가 4 명으로 나타났다. 제 1 유 
형으로 분류된 대표 진술문과 표준점수는 Table 5에서 제시하 는 바와 같다.

제 1 유형으로 분류된 대상자들이 가장 높은 긍정적 동의 를 보인 진술문을 보면 “나는 유아교육기관 현장의 과학기기 및 장비의 부족으로 인해 과학 활동을 진행하기 어렵다.” $(z=$ .778)였고, 가장 낮게 동의한 진술문은 "나는 과학적 용어나 지식을 이해하는 것에 부담을 느낀다." $(z=-1.095)$ 로 나타났다. 이 유형의 전형인 8 번이 가장 동의한 진술문은 “나는 유아교 육기관 현장의 과학기기 및 장비의 부족으로 인해 과학 활동 을 진행하기 어렵다.", "나는 동.식물 및 과학 자료 관리의 어 려움으로 인해 과학 활동을 진행하는 것이 부담스럽다.” 이었 다. 반면에 가장 부정적인 동의를 보인 진술문은 "나는 과학적 용어나 지식을 이해하는 것에 부담을 느낀다.”, “나는 누리교 육과정 중 자연탐구영역의 주제들이 어떻게 서로 연관되어 있 는지 이해하기 어렵다.”이었다. 이러한 결과로 제 1 유형의 경 우에는 유아과학교육의 특성 또는 과학적 용어나 지식을 이해 하는 것에 부담을 느끼지는 않지만 과학 환경구성 및 자료의 부족의 과학교육을 부담스러워 하는 교사들인 것으로 파악할 수 있다. 따라서 '과학 환경구성 및 자료준비에 따른 불안형'으 로 명명하였다.

제2유형: 과정과 탐구기술 중심의 과학교수방 법에 대한 불안형

제 2 유형으로 분류된 대상자는 12 명으로 나타났다. 경력이 5 년 미만인 교사가 7명, 5년-10년 미만인 교사가 2명, 10 년 이 상인 교사가 3명이었고, 기관유형은 국공립유치원 교사가 6 명, 사립유치원 교사가 6명이었으며, 소지자격은 유치원 2급 정교사가 8 명, 유치원 1 급 정교사가 3 명, 원감 자격증을 소지 한 교사가 1 명으로 나타났다. 제 2 유형으로 분류된 대표 진술 문과 표준점수는 Table 6에서 제시하는 바와 같다.

제 2 유형으로 분류된 대상자들이 가장 높은 긍정적 동의를 보인 진술문을 보면 “나는 유아들에게 실험의 결과보다는 과
정에서의 토의와 의견교환을 장려하는 것이 부담스럽다." $(z=$ .760)였고, 가장 낮게 동의한 진술문은 “나는 유아교육기관 현 장의 과학기기 및 장비의 부족으로 인해 과학 활동을 진행하 기 어렵다.” $(z=-.574)$ 이었다. 이 유형의 전형인 13 번이 가장 동의한 진술문은 "나는 유아들에게 실험의 결과보다는 과정 에서의 토의와 의견교환을 장려하는 것이 부담스럽다.”, “나 는 유아과학교육 활동의 계획과정에 유아를 참여시키는 것이 어렵다.” 이었다. 반면에 가장 부정적인 동의를 보인 진술문 은 "나는 유아가 과학은 유아가 이해하기에 너무나 어려워서 교육을 하기 어렵다고 생각한다.", "나는 과학적인 문제에 대 해 유아들이 호기심을 갖고 있지 않다고 생각한다.”이었다. 이 러한 결과는 제 2 유형의 교사들이 유아과학교육의 중요성은 잘 인식하고 있고, 유아들의 과학적 호기심에는 긍정적인 반 면에, 탐구과정에서 토의와 의견교환을 어떻게 진행해야 하 는지 부담스러워하고 탐구기술에 대한 시범을 어떻게 보여야 하는지 어려워함을 보여준다. 따라서 제 2 유형을 '과정과 탐구 기술 중심의 과학교수방법에 대한 불안형'으로 구분하여 명 명하였다.

제3유형: 유아과학교육 특성 이해부족에 따른 불안형

제 3 유형으로 분류된 대상자는 10 명으로 나타났다. 경력이 5 년 미만인 교사가 6명, 5년-10년 미만인 교사가 3명, 10년 이 상인 교사가 1 명이었고, 기관유형에서는 사립유치원 교사가 10 명이었으며, 소지자격은 유치원 2 급 정교사가 7 명, 유치원 1 급 정교사가 2 명, 원장 자격증을 소지한 교사가 1 명으로 나 타났다. 제 3 유형으로 분류된 대표 진술문과 표준점수는 Table 7에서 제시하는 바와 같다.

제3유형으로 분류된 대상자들이 가장 높은 긍정적 동의를 보인 진술문으로는 "나는 일반과학과 유아를 위한 과학이 어 떻게 다른지 이해하기 어렵다.” $(z=.873)$ 였고, 가장 낮게 동의

Table 3

Explanatory Power of Factors

\begin{tabular}{lccc}
\hline & Eigen value & Variance & Cumulative \\
\hline Type 1 & 5.184 & 15.708 & 15.708 \\
Type 2 & 4.465 & 13.531 & 29.239 \\
Type 3 & 2.871 & 8.700 & 37.939 \\
Type 4 & 2.374 & 7.195 & 45.134 \\
\hline
\end{tabular}


Table 4

P Sample Weighting Factors for Each Type

\begin{tabular}{|c|c|c|c|c|c|c|c|c|c|c|}
\hline & No. & Id & Age & Sex & $\begin{array}{l}\text { Educational experience } \\
\text { (years . months) }\end{array}$ & $\begin{array}{l}\text { Last school } \\
\text { graduation }\end{array}$ & $\begin{array}{l}\text { Institution } \\
\text { type }\end{array}$ & Certification & Class & $\begin{array}{l}\text { Factor } \\
\text { weight }\end{array}$ \\
\hline \multirow{10}{*}{$\begin{array}{l}\text { Type } 1 \\
(n=11)\end{array}$} & 1 & P8 & 38 & Female & 9.7 & Master & Public & KTG 1 & 5 & 1.93860 \\
\hline & 2 & P28 & 29 & Female & 8.7 & University & Private & KTG 1 & 4 & 1.87928 \\
\hline & 3 & P27 & 38 & Female & 13.7 & University & Public & KTG 1 & 5 & 1.73442 \\
\hline & 4 & P30 & 24 & Female & 1.7 & University & Private & KTG 2 & 4 & 1.52228 \\
\hline & 5 & P34 & 24 & Female & 1.6 & University & Public & KTG 2 & $\operatorname{mix}$ & 1.52116 \\
\hline & 7 & P7 & 22 & Female & 0.7 & College & Private & KTG 2 & 3 & .78357 \\
\hline & 8 & P19 & 23 & Female & 1.0 & College & Private & KTG 2 & 3 & .70969 \\
\hline & 9 & P29 & 26 & Female & 4.7 & University & Public & KTG 2 & 4 & .60386 \\
\hline & 10 & P17 & 27 & Female & 5.8 & College & Private & KTG 2 & 4 & .14431 \\
\hline & 11 & P32 & 26 & Female & 2.7 & University & Public & KTG 2 & 4 & -.18064 \\
\hline & 15 & P33 & 28 & Female & 3.7 & University & Public & KTG 2 & 4 & 1.42041 \\
\hline & 16 & $\mathrm{P} 40$ & 39 & Female & 15.7 & University & Public & KTG 1 & 5 & 1.42041 \\
\hline & 17 & P26 & 37 & Female & 13.7 & College & Private & $\mathrm{AD}$ & 5 & 95509 \\
\hline & 18 & P38 & 48 & Female & 7.7 & University & Public & KTG 2 & 3 & .55878 \\
\hline & 19 & P11 & 30 & Female & 7.4 & University & Private & KTG 1 & 3 & .35272 \\
\hline & 20 & P3 & 27 & Female & 3.7 & University & Private & KTG 2 & 4 & .34798 \\
\hline & 21 & $\mathrm{P} 23$ & 26 & Female & 2.5 & University & Public & KTG 2 & 4 & .23286 \\
\hline & 22 & P25 & 26 & Female & 4.7 & College & Private & KTG 2 & 5 & .20723 \\
\hline & 23 & P5 & 26 & Female & 3.7 & University & Private & KTG 2 & 4 & -.66752 \\
\hline \multirow{4}{*}{$\begin{array}{l}\text { Type } 3 \\
(n=10)\end{array}$} & 24 & P12 & 38 & Female & 8.10 & Master & Private & KTG 1 & 3 & 2.23773 \\
\hline & 31 & P22 & 24 & Female & 3.7 & College & Private & KTG 2 & 3 & -.06154 \\
\hline & 32 & P15 & 24 & Female & 1.0 & College & Private & KTG 2 & 3 & -.15373 \\
\hline & 33 & P10 & 50 & Female & 27.0 & University & Private & $\mathrm{D}$ & - & -.84514 \\
\hline \multirow{7}{*}{$\begin{array}{l}\text { Type } 4 \\
(n=7)\end{array}$} & 34 & P37 & 32 & Female & 7.9 & Master & Public & KTG 2 & 3 & 2.38867 \\
\hline & 35 & P39 & 41 & Female & 20.0 & University & Public & KTG 1 & 5 & 1.81602 \\
\hline & 36 & P35 & 23 & Female & 0.8 & University & Public & KTG 2 & 5 & 1.29182 \\
\hline & 37 & P9 & 24 & Female & 2.2 & College & Private & KTG 2 & 3 & 1.25263 \\
\hline & 38 & P36 & 34 & Female & 1.7 & University & Public & KTG 2 & $\operatorname{mix}$ & 1.19129 \\
\hline & 39 & P21 & 24 & Female & 2.9 & College & Private & KTG 2 & 3 & .20891 \\
\hline & 40 & P20 & 23 & Female & 1.7 & College & Private & KTG 2 & 4 & -.20881 \\
\hline
\end{tabular}

Note. $\mathrm{D}=$ director; $\mathrm{AD}=$ assistant director; KTG 1 = kindergarten teacher grade 1 ; KTG 2 = kindergarten teacher grade 2.

한 진술문은 “나는 유아들이 읽기, 쓰기, 능력을 갖추기 전에 는 과학을 가르치기 어렵다고 본다.” $(z=-.845)$ 로 나타났다. 이 유형의 전형인 12 번이 가장 동의한 진술문은 "나는 과학에 필
요한 탐구기술이 무엇인지 명확히 이해하기 어렵다.", "나는 과학적 탐구기술이나 호기심을 기르는 것이 유아들에게 중요 하지 않다고 생각한다.” 이었다. 반면에 가장 부정적인 동의를 
Table 5

Typical Statements and Standard Score for Type 1

\begin{tabular}{cll}
\hline No. & \multicolumn{1}{c}{ Q statements } & $Z$-score \\
\hline 30 & I am afraid of proceeding science activities because of a lack of scientific equipment in my institution. & .778 \\
32 & I am afraid of proceeding science activities because of difficulties in taking care of animals, plants and science materials. & .769 \\
21 & I think it is too early to teach science for preschoolers. & .739 \\
33 & I am afraid of proceeding science activities because my classroom gets noisy and massy. & .707 \\
22 & I think science education happens naturally without careful planning of science activities. & .530 \\
4 & I have difficulty understanding cognitive development of children related to science education. & -.685 \\
1 & I have difficulty understanding differences between science for children and general science. & -.800 \\
5 & I have difficulty understanding how the topics of Nature inquiry area in Nuri curriculum are inter-related. \\
2 & I have difficulty clearly understanding scientific inquiry skills for children. & -.913 \\
3 & I feel burdened by learning scientific terms and knowledge. & -1.003 \\
\hline
\end{tabular}

Table 6

Typical Statements and Standard Score for Type 2

\begin{tabular}{clll}
\hline No. & & Q statements & $Z$-score \\
\hline 14 & I have difficulty emphasizing discussion and exchanging ideas in process rather than focusing on results in science activities. & .760 \\
10 & I have difficulty demonstrating scientific inquiry skills such as observation, test, and prediction. & .524 & .505 \\
9 & I have difficulty providing developmentally appropriate science materials for young children. & .495 & .428 \\
19 & I have difficulty including children when I plan science activities. & -.501 \\
24 & I think socio-emotional activities are more important than science activities. \\
33 & I am afraid of proceeding science activities because my classroom gets noisy and massy. \\
21 & I think it is too early to teach science for preschoolers. & \\
17 & I think children are not interested nor have curiosity in the scientific problems. \\
27 & I think basic principles regarding object and matter are not important for children. & -.554 \\
30 & I am afraid of proceeding science activities because of a lack of scientific equipment in my institution. & -.558 \\
\hline
\end{tabular}

Table 7

Typical Statements and Standard Score for Type 3

\begin{tabular}{clll}
\hline No. & & Q statements & $Z$-score \\
\hline 1 & I have difficulty understanding differen ces between science for children and general science. & .873 \\
2 & I have difficulty clearly understanding scientific inquiry skills for children. & .845 & .814 \\
10 & I have difficulty demonstrating scientific inquiry skills such as observation, test, and prediction. & .702 & .687 \\
5 & I have difficulty understanding how the topics of Nature inquiry area in Nuri curriculum are inter-related. & -.411 \\
12 & I have difficulty planning step by step instruction for science activities. & -.485 \\
19 & I have difficulty including children when I plan science activities. & I have difficulty finding adequate questions for developing scientific concepts of young children. \\
18 & I think individually appropriate science education for young children is difficult. \\
14 & I have difficulty emphasizing discussion and exchanging ideas in process rather than focusing on results in science activities. & -.592 \\
15 & I think teach young children science is difficult if they cannot read or write yet. & -.845 \\
\hline
\end{tabular}


보인 진술문은 "나는 유아들이 읽기, 쓰기, 능력을 갖추기 전 에는 과학을 가르치기 어렵다고 본다.", “나는 유아들에게 과 학교육을 강조하는 것은 너무 이르다고 생각한다.”이었다. 이 러한 결과는 제 3 유형의 교사들이 유아기 과학교육 실시의 필 요성은 잘 알고 있으나, 유아과학교육이 일반과학과 어떻게 다른지, 유아과학교육에서 강조하는 탐구기술이나 통합적 접 근을 이해하는데 어려움을 겪는 것을 의미한다. 따라서 제 3 유 형을 ‘유아과학교육 특성 이해부족에 따른 불안형'으로 구분 하여 명명하였다.

\section{제 4 유형: 단순 과학 기피형}

제4유형으로 분류된 대상자는 7명으로 나타났다. 경력이 5년 미만인 교사가 5명, 5년-10년 미만인 교사가 1명, 10년 이상 인 교사가 1 명이었고, 기관유형에서는 국공립유치원 교사가 4 명, 사립유치원 교사가 3 명이었으며, 소지자격은 유치원 2 급 정교사가 6 명, 유치원 1 급 정교사가 1 명으로 나타났다. 제 4 유 형으로 분류된 대표 진술문과 표준점수는 Table 8에서 제시하 는 바와 같다.

제4유형으로 분류된 대상자들이 가장 높은 긍정적 동의를 보인 진술문으로는 "나는 과학적 용어나 지식을 이해하는 것 에 부담을 느낀다." $(z=1.082)$ 였고, 가장 낮게 동의한 진술문 은 "나는 과학 활동을 지도하는 것보다 기본생활습관을 지도 하는 것이 중요하다고 생각한다.” $(z=-1.210)$ 였다. 이 유형의 전형인 37번이 가장 동의한 진술문은 "나는 과학적 용어나 지 식을 이해하는 것에 부담을 느낀다.", “나는 유아과학교육 활 동의 경험이 다른 영역에서의 경험과 연계될 수 있도록 통합
하여 진행하는 것이 어렵다.”이었다. 반면에 가장 부정적인 동 의를 보인 진술문은 "나는 유아들이 읽기, 쓰기, 능력을 갖추 기 전에는 과학을 가르치기 어렵다고 본다.”, “나는 유아들에 게 과학교육을 강조하는 것은 너무 이르다고 생각한다.”이었 다. 제 4 유형의 경우에 과학교육 자체에 대한 부담과 기피가 교 사와 유아자신을 대상으로 총체적으로 나타났다. 따라서 과학 교육 자체에 대해 불안을 느끼는 집단인 '단순 과학 기피형'으 로 명명하였다.

\section{논의 및 결론}

본 연구는 유아교사들의 유아과학교육에 대한 불안이 어떻게 유형화되는지 Q 방법론을 통해 분석하여, 집단이 가진 고유 한 불안인식의 특성을 파악하고, 현장에 도움이 될 수 있는 구 체적인 제언을 도출하기 위해 설계되었다. 이를 위해 일차적 으로 유아 과학교육과 관련된 전문 서적, 연구 논문 등을 통해 268 개의 Q 모집단을 구성하고, 현장 전문가와 학계 전문가의 도움을 얻어 33 개의 최종 Q 진술문을 선정하였다. 이후 $\mathrm{P}$ 표 본으로 선정된 서울, 경기지역의 유아교사 40 명에게 33 문항 에 대한 주관적인 동의 정도를 9점 강제분류방식에 의거하여 $\mathrm{Q}$ 분류 하도록 하였다.

수집된 자료를 QUANL program으로 통계 처리하여 분 석한 결과, 과학교육과 관련하여 가지는 주관적 불안의 유 사한 정도에 따라 유아교사들은 4 개의 유형으로 분류되었 다. 4 개의 유형은 전체변량의 $45.13 \%$ 를 설명하고 있는데 H.S. Park (2007)에 따르면 일반적으로 누적 설명량이 $40 \%$ 이상

Table 8

Typical Statements and Standard Score for Type 4

\begin{tabular}{clr}
\hline No. & & Q statements \\
\hline 3 & I feel burdened by learning scientific terms and knowledge. & 1.082 \\
16 & I think science is too difficult for children so that it is hardly teachable. & .907 \\
13 & I have difficulty integrating science activities across the other educational areas. & .709 \\
8 & I have difficulty finding adequate questions for developing scientific concepts of young children. & .555 \\
10 & I have difficulty demonstrating scientific inquiry skills such as observation, test, and prediction. \\
22 & I think science education happens naturally without careful planning of science activities. \\
26 & I think developing curiosity in science and inquiry skills are not important for children. \\
28 & I think basic knowledge regarding object and matter is not important for children. \\
24 & I think socio-emotional activities are more important than science activities. \\
23 & I think teaching basic self-help skills are more important than proceeding scientific activities. & -.571 \\
\hline
\end{tabular}


이면 의미를 가진다고 하였다. 각 유형별 설명력은 제 1 유형 이 $15.70 \%$, 제 2 유형이 $13.53 \%$, 제 3 유형이 $8.70 \%$, 제 4 유형이 $7.19 \%$ 였다. 본 연구에서 도출된 유형들의 특징을 대표진술문 과 전형적 응답자를 중심으로 분석하여, 제 1 유형은 과학 환경 구성 및 자료준비에 따른 불안형, 제 2 유형은 과정과 탐구기 술 중심의 과학교수방법에 대한 불안형, 제 3 유형은 유아과학 교육 특성 이해부족에 따른 불안형, 제 4 유형은 단순 과학 기 피형으로 명명하였다. 본 연구에서 얻어진 4 개의 유형에 대해 논의하면 다음과 같다.

첫째, 과학 환경구성 및 자료준비에 따른 불안형으로 명명 된 제 1 유형의 교사들은 유아과학교육과 관련된 인지발달개 념이나 유아과학교육이 가지는 일반과학과의 차별성은 잘 이 해하고 있으나, 환경구성의 어려움 또는 자료 부족으로 인하 여 어려움을 겪고 있는 집단이다. 유아교사들이 과학관련 환 경구성과 재료로 인한 어려움을 겪는다는 것은 여러 차례 보 고된 바 있다. 예로서 K. M. Lee (2005)는 과학교육을 여러 영 역으로 세분해서 불안감의 상대적 점수를 파악하였는데 유아 교사들의 자료준비와 관련된 점수가 가장 높았다. 과학 환경 구성 및 자료와 관한 불안을 해소하기 위해서는 충분한 재원 을 확보하여 자료를 공급해 주는 것이 일차적인 해결책이 될 수 있으나, 본 연구에서는 다른 시각으로 이 현상을 접근해 보 고자 한다. 시중에 판매되는 유아과학교구들은 주로 단순한 실험 몇 가지를 수행할 수 있는 자료들로 구성이 되어 있어서 활용성이 떨어지며 한번 구입하면 보관하고 관리해야 하는 교사의 부담이 따른다. 사실 유아과학교육은 통합적으로 진 행되어야 하고, 과학 영역에서만 일어나는 것이 아니며, 호기 심을 가지고 일상생활의 경험들을 탐구하는 과정에서 과학적 태도와 능력을 길러주는 것이기 때문에 일반과학에 적용되는 특별한 환경구성이나 자료가 많이 필요한 것은 아니다(Chaille \& Britain, 1991), 따라서 환경구성과 자료부족에 관한 유아교 사의 불안을 해소하기 위해서는 물질적 지원보다는 현장에서 유아과학교육은 반드시 특정한 과학 자료가 있어야 한다는 편견이 존재하는지 점검해 보고, 교사가 일상생활에서 과학 교육에 적용할 만한 환경이나 자료를 찾아내는 능력을 갖추 는 것이 필요하다.

둘째, 제 2 유형의 교사들은 과정과 탐구기술 중심의 과학교 수방법에 대한 불안형으로 과학교육에 활용할 만한 환경구성 및 자료준비에 대한 불편함은 크게 지각하지 못하고, 물체와 물질을 탐구하는 것이 유아들에게 의미 있는 것임은 알고 있 으나, 탐구과정을 어떻게 이끌어가야 하는지, 탐구기술을 유 아들에게 어떻게 시범 보여야 하는지에 어려움을 겪고 있었
다. 결과를 강조하는 교육은 가시적 결과물이 존재하고 그 결 과물에 따라 교육적 평가가 이루어지나, 과정에 가치를 두는 교육은 그 과정에서 일어나는 여러 가지 사건과 현상을 표상 하고 이를 상호 교환하는 것에 가치를 둔다(J. H. Lee, 1998). 그러나 유아들은 탐구 과정 속에서 일어나는 여러 가지 경험 에 대한 의미 있는 표상을 외부적으로 상징화하여 상호교환 하기 어렵다. 이럴 경우, 유아들이 내적 표상을 상징화하고 이 미지화 하여 교환할 수 있도록 돕는 매우 전문적인 교사의 역 할이 요구되는데 이는 고도의 전문성을 요구하며, 실패할 경 우 교사는 자신의 역량을 낮게 지각할 수 있으며, 과학교육이 실패했다고 느낄 수 있다.

한편, 유아과학에서 요구되는 탐구기술은 일반과학(사회 과학 및 자연과학)에서 사용되는 탐구기술과 크게 다르지 않 다(Chaille \& Britain, 1991). 탐색, 관찰, 측정 등을 통해 기초 자료를 수집하고, 비교와 분류를 통해 가설을 발견하며, 예측 과 가설을 실험해보고, 가설을 수용하거나 수정하는 일련의 순환과정은 비록 다루어지는 재료와 도구가 다를지라도 성인 과학의 연구기술과 매우 닮아있다. Piaget에 의하면 이는 일련 의 도식 발달과정이며, 조절과 동화, 인지적 갈등 및 균형화가 포함된 순환적 과정이다(Tsou, 2006). 문제는 많은 교사들이 탐구기술이 무엇인지 잘 이해하지 못하고 있으며 스스로도 별로 활용해 본 경험이 없다는 것이다. 누리과정의 자연탐구 영역에서 직접 언급한 탐구기술은 총 네 가지(탐색, 관찰, 비 교, 예측)이지만(Ministry of Education and Science Technology, 2013), 실제로 유아들이 탐구과정에서 사용하는 탐구기술의 수는 이것보다 훨씬 다양할 뿐더러, 교사용 지도서나 해설서 에도 네 가지의 탐구기술에 대한 명확하고 구체적인 설명이 제시되어 있지 않다. 따라서 교사 본인이 경험하고 체득하지 못한 것을 유아들에게 적절히 지도하지 못하는 것은 당연하 다.

유아과학교육의 교수방법과 관련된 핵심적이고 고차원적 인 부분에 어려움을 겪고 있는 제 2 유형의 교사들은 오히려 유 아과학교육의 본질에 대한 고민을 가장 많이 한 교사들로 간 주될 수 있다. 이들에게 도움을 주기 위해서는 유아들이 탐구 과정에서 표상하는 것들을 외부적으로 상징화 하거나 이미지 화 하도록 돕는 다양한 전략을 구체적으로 알려주는 것이 필 요하다. 탐구기술과 관련해서는 국가수준의 교육과정에서 유 아들이 활용할 수 있는 탐구기술들은 무엇이며, 각 탐구기술 의 기능은 무엇이며 탐구의 어느 단계에 사용될 수 있는지를 명확하고 구체적으로 규정한 후에, 유아교사들이 이러한 과 정을 먼저 경험하고 습득하면, 자연스럽게 유아교육 현장에 
서 성공적으로 적용될 수 있을 것이다.

셋째, 제 3 유형은 유아과학교육 특성 이해부족에 따른 불안 형으로 이 교사들은 일반과학과 유아과학의 차이점 및 탐구 기술에 대한 명확한 이해를 어렵게 인식함에도 불구하고 과 학을 가르치는 것은 어렵지 않다는 모순된 인식성향을 보인 다. 제 3 유형 교사들은 유아과학교육의 특성을 제대로 살리지 못하고 다른 영역의 교과와 별 차이 없이 교수하면서도 특별 히 잘못된 점을 인식하지 못할 가능성이 큰 유형이다. 이 유형 의 교사들을 위해서는 유아과학교육의 목적은 호기심을 유지 하면서 탐구하는 태도와 능력을 기르는 데 있으며(Gelman \& Brenneman, 2004), 비록 자료와 도구는 다르지만 성인과학과 유사한 탐구기술을 사용하는 것(Hong \& Diamond, 2012)이 특 징이라는 것을 재개념화 할 수 있는 기회를 제공하여야 한다.

넷째, 제4유형은 단순 과학 기피형으로 유아과학교육이 필 요한 것은 알고 있으나 교사 자신이 과학을 자신 없어하고 어 려워하며, 이러한 부정적인 인식으로 인해 유아과학교육의 필요성마저 잠식당한 유형으로 볼 수 있다. H.-S. Cho와 Yoo (2011)은 교사들이 다양한 과학 활동을 경험하고 교사 스스로 가 과학이 재미있다는 감동을 느낄 때, 유아들에게도 과학수업 을 하고자 하는 의욕이 생긴다고 하였다. 이 교사들에게 가장 필요한 것은 과학에 대한 교사의 자신감 함양이다. 누구나 좋 아하거나 관심 있어 하는 과학 분야가 하나 정도는 반드시 존 재하기 때문에 그 곳에서부터 시작하여 점차 과학에 대한 인식 을 개선하고자 하는 지속적인 노력과 자기반성이 요구된다.

종합하면, Q 방법론을 활용하여, 유아교사들의 과학교육 과 관련하여 인식하는 불안을 유형화 해 본 결과 환경구성 및 자료준비, 교수방법, 유아과학교육 특성 이해부족, 그리고 단 순과학 기피 등으로 표출되었다. 심층적으로는, 이러한 불안 인식이 대체로 유아과학교육이 가진 독특함으로 인해 특별 한 도전이 유아교사들에게 주어졌기 때문으로 해석된다. 일 반교과로 인식되는 과학을 통합적으로 적용하면서 흥미 있는 경험들을 일상생활에서 발굴하여 과학 자료로 활용해야 하는 것은 교수방법의 영역이며, 탐구과정에서 발생하는 유아의 표상을 외부적으로 상징화 하도록 도와서 이를 상호교환하 게 끔 하는 것은 인식론과 인지심리학의 영역이다. 탐색, 자료 수집, 가설수립, 검증의 단계에 사용되는 탐구기술을 이해하 고 시범보이는 것은 자연과학 혹은 사회과학의 연구방법론의 영역이라 하겠다. 유아과학교육은 이러한 다양한 분야의 전 문기술이 요구되는 것으로서 단순히 자연과학의 유아용 축소 판이라고 보기 어렵다. 따라서 유아과학교육과 관련된 교사 의 불안은 해소하기 위해서는 단순히 직전교육과 현장연수의
양을 확대하는 것을 떠나서 국가수준 교육과정이나 교사교육 주체가 유아과학교육의 본질을 제대로 분석하고 이에 대한 교육과정을 구체적으로 구성하는 것이 그 출발점이 될 수 있 겠다.

본 연구의 제한점은 다음과 같다. 첫째, $\mathrm{Q}$ 방법론과 관련된 것으로 $\mathrm{Q}$ 모집단의 대표성과 $\mathrm{Q}$ 표본의 포괄성에 한계가 분명 히 존재한다. 본 연구는 서울 및 경기도에 거주하는 유아교사 40 명을 대상으로 과학교육과 관련된 불안의 주관성을 파악하 였다. 따라서 전체 유아 교사의 인식을 대표하는데 어려움이 있다. 또한 이 연구에서 수집한 Q 모집단의 진술문이 과학 불 안에 대한 모든 의견이나 생각을 담고 있다고 할 수 없기에 Q 표본의 포괄성에 한계가 있음을 밝힌다.

둘째, 본 연구에서 파악된 네 가지 유형의 과학 불안 유형 이 응답자의 $45.13 \%$ 만을 설명해주는 데 그쳤다. 일반적으로 인간행동의 복잡성 때문에 누적된 분산이 $25 \%$ 를 넘으면 설 명력을 가지고, 누적된 설명량이 $40 \%$ 면 의미가 있다고 보기 때문에 본 연구의 설명력이 문제가 있는 것은 아니지만, 유아 교사의 과학 불안에 대한 추가적인 유형이 존재할 가능성이 있다. 따라서 향후 연구에서는 추가적인 과학 불안 내용을 도 출하여 유형을 확장하는 연구로 보완될 수 있기를 기대한다.

\section{Notes}

This article is a part of the first author's master's thesis submitted in 2016.

\section{Conflict of Interest}

No potential conflict of interest relevant to this article was reported.

\section{References}

\section{In English}

Chaille, C., \& Britain, L. (1991). The young child as scientist: A constructivist approach to early childhood science education. New York: Harper Collins.

Gelman, R., \& Brenneman, K. (2004). Science learning pathways for young children. Early Childhood Research Quarterly, 19(1), 150-158. 
Hong, S.-Y., \& Diamond, K. E. (2012). Two approaches to teaching young children science concepts, vocabulary, and scientific problem-solving skills. Early Childhood Research Quarterly, 27(2), 295-305.

Perry, G., \& Rivkin, M. (1992). Teachers and science. Young Children, 47(4), 9-16.

Sherwood, R. D., \& Westerback, M. E. (1983). A factor analytic study of the state trait anxiety inventory utilized with preservice elementary teachers. Journal of Research in Science Teaching, 20(3), 225-229.

Tsou, J. Y. (2006). Genetic epistemology and Piaget's philosophy of science Piaget vs. Kuhn on scientific progress. Theory and Psychology, 16(2), 203-224.

\section{In Korean}

Cho, B. K., \& Go, Y.-M. (2004). A study on factors influencing kindergarten teachers' science anxiety. Journal of the Korean Association for in Science Education, 24(2), 267-276.

Cho, B. K., Go, Y.-M., \& Nam, O. J. (2015). Science in early childhood education. Paju: Yangseowon.

Cho, B. K., \& Seo, S. Y. (2001). Self-efficacy beliefs of kindergarten science teachers. Korean Journal of Child Studies, 22(2), 361-373.

Cho, H.-S. (1998). Early childhood teachers' self-efficacy in teaching science. Korean Journal of Early Childhood Education, 18(2), 283-301.

Cho, H.-S., \& Yoo, E. Y. (2011). Elements of good science teaching as perceived by early childhood teachers with high science teaching self-efficacy. Korean Journal of Early Childhood Education, 31(2), 333-359.

Cho, J. H., \& Kim, K. S. (2013). Early childhood teachers' science teaching intentions and science anxiety based on their level of pedagogical content knowledge in science teaching. The Journal of Eco-Early Childhood Education, 12(1), 139-158.

Han, Y. M. (2010). Early childhood science education. Seoul: Changjisa.

Hwang, U. M., \& Cho, H. S. (2001). (Research to improve the ability for) early childhood science education. Paju: Jungminsa.

Kang, K.-S. (2007). Science anxiety of science-gifted elementary school students (Unpublished master's thesis). Korea National University of Education, Cheongju, Korea.

Kim, B. J. (1999). Consumer taxonomy using Q methodology. Korea Marketing Review, 14(4), 53-71.

Kim, H. (2013). Effect on early childhood teachers' self-efficacy in teaching science of attitude toward science and knowledge about science education. Korean Journal of Early Childhood Education, 33(2), 281-296.

Kim, H. \& Chae, J. Y. (2014). The influence of educare teachers' science education knowledge and attitudes towards science on science teaching anxiety. Journal of Korean Child Care and Education, 10(3), 69-84. doi:10.14698/ jkcce.2014.10.3.069

Kim, H. K. (2008). Q methodology: Philosophy, theories, analysis, and application. Seoul: Communication Books.

Kim, M. A. (2002). The effects of inquiry-centered science education based on constructivism for preservice early childhood teachers (Unpublished doctoral dissertation). Seoul Women's University, Seoul, Korea.

Kim, S. E. (2010). Theory and philosophy of Q methodology. Korean Society and Public Administration, 20(4), 1-25.

Kim, Y. H. (2010). Prospective early childhood educators and early childhood special educators' self-reported instructional competencies in the 7 th national preschool curriculum. The Korean Journal of Early Childhood Special Education, 10(2), 139-159.

Kim, Y.-O., Lee, G.-R., Cho, H.-J., \& Cha, G.-A. (2012). A study on early childhood teachers' science activity anxiety, attitude, and teaching efficacy according to their scientific knowledge levels. The Journal of Korea Open Association for Early Childhood Education, 17(1), 99-115.

Kwon, J. Y. (2007). Study of science education practices based upon science teaching efficacy belief levels of kindergarten teachers. The Journal of Eco-Early Childhood Education, 6(1), 85-113.

Lee, E.-J. (2010). Development of an early childhood science education lecture model for enhancing pedagogical content knowledge in pre-service teachers' course (Unpublished doctoral dissertation). Korea National University of Education, Cheongju, Korea.

Lee, E. J. (2013). Relationships between pedagogical content knowledge in science teaching of pre-service early childhood teachers and their attitude toward science and science teaching efficacy belief. Journal of Korean Child Care and Education, 9(4), 135-158.

Lee, J. H. (1998). Concept of representation: Its relation to symbol, image, and imitation. Korean Journal of Early Childhood Education, 18(1), 55-68.

Lee, K. M. (2005). An investigation of science anxiety of kindergarten teachers. Journal of Future Early Childhood Education, 12(1), 385-406.

Lee, K. M. (2009). Teachers' perception on their own science teaching. Early Childhood Education Research \& Review, 13(3), 237-263.

Lee, M.-A. (2008). A study on the child care teacher's perception of early childhood science education (Unpublished master's thesis). Inje University, Gimhae, Korea.

Lee, S. J. (2007). A study on the child care teacher's goal of scientific education and understanding as well as practice teaching 
method (Unpublished master's thesis). Duksung Women's University, Seoul, Korea.

Ministry of Education and Science Technology. (2013). Manual book of Nuri curriculum for the 3 to 5 years old. Seoul: Ministry of Education and Science Technology.

Ministry of Health and Welfare. (2013). The third standard care program. Sejong: Ministry of Health and Welfare.

Park, H.-S. (2007). Analysis of inquiry levels in the laboratory practices by science teaching efficacy belief of elementary school teachers (Unpublished master's thesis). Korea National University of Education, Cheongju, Korea.

Park, S. (2003). Development of PCK (pedagogical content knowledge) instrument in science teaching for elementary school teachers. The Journal of Korean Teacher Education, 20(1), 105-134.

QUANL program [Computer software]. Retrieved from Korean Society for Scientific Study of Subjectivity website: http:// www.kssss.org

Shin, D. (2010). The effect of practical teaching for developing in prospective secondary science teachers' science teaching efficacy belief and pedagogical content knowledge (Unpublished master's thesis). Ewha Womans University, Seoul, Korea.

Shin, E.-S., \& Kim, H.-J. (2007). Development of assessment tools for kindergarten teachers' pedagogical content knowledge of science teaching. The Journal of Korean Teacher Education, 24(3), 221-250.

\section{ORCID}

Ja Eun Kim

http://orcid.org/0000-0001-5962-0325

Yeon Ha Kim http://orcid.org/0000-0001-5547-2900

Received February 29, 2016

Revision received June 10, 2016

Accepted June 10, 2016 